\title{
Physics of a microsystem starting from non-equilibrium quantum statistical mechanics
}

\author{
L. Lanz ${ }^{1}$, O. Melsheimer ${ }^{2}$ and B. Vacchini ${ }^{1}$ \\ ${ }^{1}$ Dipartimento di Fisica dell'Università di Milano and INFN, Sezione di Milano, \\ Via Celoria 16, I-20133, Milan, Italy \\ 2 Fachbereich Physik, Philipps-Universität, Renthof 7, D-35032, Marburg, Germany \\ (e-mail: lanz@mi.infn.it; melsheim@mailer.uni-marburg.de; vacchini@mi.infn.it)
}

November 1, 2018

\begin{abstract}
In this paper we address the problem to give a concrete support to the idea, originally stemming from Niels Bohr, that quantum mechanics must be rooted inside the physics of macroscopic systems. It is shown that, starting from the formalism of the non-equilibrium statistical operator, which is now a consolidated part of quantum statistical mechanics, particular correlations between two isolated systems can be singled out and interpreted as microsystems. In this way also a new framework is established in which questions of decoherence can be naturally addressed.

Keywords: foundations of quantum mechanics; decoherence; non-equilibrium statistical mechanics
\end{abstract}

\section{Introduction}

The motivation of this paper is twofold: on the one side the question about foundations of quantum mechanics still catches the interest [1] of many scientists, mainly due to important improvements in experimental physics and to the increasing relevance of the concept of entanglement in the realm of quantum computation; on the other side there is relevant progress in the theory of non-equilibrium statistical mechanics [2]. That a deep connection between these two apparently unrelated subjects exists should be clear if one thinks about the way Bohr discussed foundations of quantum mechanics, facing Einstein's objections against quantum mechanics at the beginning of all questions about reality of microsystems. Let us recall that Bohr's main concern was the very rooting of microsystems inside objectively given macroscopic reality. This point of 
view has been pursued further by Ludwig [3], who starting from a purely phenomenological macroscopic setting was able to derive and extend the formalism of quantum mechanics, giving an insight into what should be understood as reality of microsystems. In this connection also a more profound theory of measurement, with respect to which textbook measurement theory appears as a very naive idealization, has grown up and has found many applications, typically in quantum optics, by the work of many researchers [4]. A very systematic and comprehensive account of all this is given by Holevo's work [5], who contributed to all relevant steps.

Despite the formal achievements of this more realistic axiomatic structure, the basic necessity of this approach and its real effectiveness to cope successfully difficulties in the foundations of quantum mechanics is still not in tune with the general idea that only the physics of elementary particles should have a fundamental role, any compelling resorting to macrophysics being only an annoying bypass. Our attitude instead is that the consideration of macroscopic physics gives a strong motivation in favor of the first standpoint: in fact any real experiment is described and realized in terms of macrosystems. The basic tool for this is quantum field theory for confined systems: the extraction of the concept of particles and of their local interactions could be a subsequent concern, to be perhaps performed using the typical procedures of a thermodynamic limit.

Obviously our attempt can only start if a working theory of non-equilibrium macrosystems exists. In this connection the situation has improved relying on the concept of relevant variables and on the method of the non-equilibrium statistical operator [2]. In previous papers some improvement in the foundations of this concept was proposed insisting on the ideas of confinement, isolation and preparation of a macrosystem [6] during some initial time interval. A rather general and tractable situation, that we called "simple dynamics", arises if the evolution of the local relevant variables is driven by two-point Kubo correlation functions involving relevant variables and their currents at points separated by a short time interval: in other words memory contributions are assumed to be restricted to short time intervals. On the contrary if two isolated macrosystems exchange a particle during the preparation stage a possibly long living correlation between the systems arises if this particle presents a coherent independent dynamics for a suitable time interval: in the present paper just this situation is discussed.

The treatment seems to us interesting for two reasons. On one side the microphysical description can be read off from the macroscopic dynamics of the system: the initial state of the microsystem can be related to a macroscopic source, the time evolution of the microsystem is part of the dynamics of the statistical operator, the measurement of an observable for the microsystem is related to the values of relevant variables of the macrosystem; obviously all this is treated only in a very schematic way. On the other side one gains a way to face the case of long lived correlations using the dynamics of the microsystem, thus providing an example in which the difficult problem of a dynamics with 
memory can be treated. A final remark is now in order; in our description a microsystem unavoidably appears having a macrosystem in the background: then its time evolution is necessarily affected by the decoherence phenomenon. Our treatment gives a natural reason to decoherence, so that is does not appear as an additional feature of quantum mechanics or as a motivation to modify the theory.

The paper is organized as follows: in Sect. 2 we introduce the statistical operator describing a system composed of two parts which are isolated from each other after the preparation procedure, but keep record of a correlation arising during the preparation time; the correction of the dynamics due to this correlation is worked out in Sect. 3 and interpreted as a microsystem produced in system 2, propagating and detected in system 1. Problems related to the dynamics of the microsystem inside a macrosystem are outlined in Sect. 4.

\section{Separation of two isolated macrosystems and initial correlations}

We rely in the following on a general theory of an isolated macroscopic system inside quantum field theory that has been proposed in previous papers [6] and is very close to statistical mechanics formulated in terms of the non-equilibrium statistical operator by Zubarev and more recently by Morozov and Roepke [2], differing only in the introduction of a "preparation procedure" which extends over a finite time interval and refers to a confined isolated system, thus avoiding in principle the thermodynamic limit at the level of foundations of macroscopic physics. According to this theory a set of relevant variables is selected, slow enough on a suitable time scale, for which the very concept of isolation can make sense, built in terms of suitable field densities. Let us take $\left[T, t_{0}\right]$ as preparation time interval, the statistical operator at time $t_{0}$ is given by

$$
\begin{aligned}
\hat{\varrho}_{t_{0}}=\exp \{ & -\sum_{j} \int d \mathbf{x} \zeta_{j}\left(\mathbf{x}, t_{0}\right) \hat{A}_{j}(\mathbf{x})+\sum_{j \alpha} \int d \mathbf{x} \gamma_{j \alpha}(\mathbf{x}) \int_{T}^{t_{0}} d t^{\prime} \hat{A}_{j}\left(\mathbf{x},-\left(t_{0}-t^{\prime}\right)\right) h_{j \alpha}\left(t^{\prime}\right) \\
& +\sum_{j \alpha} \int d \mathbf{x} \gamma_{j \alpha}(\mathbf{x}) \cdot \int_{T}^{t_{0}} d t^{\prime} \hat{\mathbf{J}}_{j}\left(\mathbf{x},-\left(t_{0}-t^{\prime}\right)\right) h_{j \alpha}\left(t^{\prime}\right) \\
& \left.-\sum_{j} \int d \mathbf{x} \zeta_{j}(\mathbf{x}, T) \hat{A}_{j}\left(\mathbf{x},-\left(t_{0}-T\right)\right)\right\},
\end{aligned}
$$

with $\zeta_{j}\left(\mathbf{x}, t_{0}\right)$ the classical state parameters such that

$$
\operatorname{Tr}\left(\hat{A}_{j}(\mathbf{x}) \hat{\varrho}_{t_{0}}\right)=\operatorname{Tr}\left(\hat{A}_{j}(\mathbf{x}) \hat{w}_{\zeta\left(t_{0}\right)}\right)
$$


where $\hat{w}_{\zeta(t)}$ is the generalized Gibbs state related to the state parameters $\zeta_{j}(t)$

$$
\hat{w}_{\zeta(t)}=\frac{\exp \left\{-\sum_{j} \int d \mathbf{x} \zeta_{j}(\mathbf{x}, t) \hat{A}_{j}(\mathbf{x})\right\}}{\operatorname{Tr}\left(\exp \left\{-\sum_{j} \int d \mathbf{x} \zeta_{j}(\mathbf{x}, t) \hat{A}_{j}(\mathbf{x})\right\}\right)} .
$$

$\hat{A}_{j}(\mathbf{x})$ are the densities of the relevant variables, associated to the currents $\hat{\mathbf{J}}_{j}(\mathbf{x})$ and $\gamma_{j \alpha}, h_{j \alpha}$ are the parameters describing the preparation procedure. We assume for simplicity that all the field variables are built with only one underlying quantum Schrödinger field $\hat{\psi}(\mathbf{x})$, satisfying

$$
\left[\hat{\psi}(\mathbf{x}), \hat{\psi}^{\dagger}\left(\mathbf{x}^{\prime}\right)\right]_{ \pm}=\delta^{3}\left(\mathbf{x}-\mathbf{x}^{\prime}\right)
$$

In this context one can very easily formalize the idea that a macroscopic system is separated into two non interacting parts 1 and 2 corresponding to two non overlapping regions $\omega_{1}, \omega_{2}$. For times $t \geq t_{0}$ let us introduce two complete sets of normal modes for both regions: $\left\{u_{n}^{(1)}(\mathbf{x}), \mathbf{x} \in \omega_{1}\right\}$ and $\left\{u_{n}^{(2)}(\mathbf{x}), \mathbf{x} \in \omega_{2}\right\}$, determined by

$-\frac{\hbar^{2}}{2 m} \Delta_{2} u_{n}^{(1)}(\mathbf{x})+V(\mathbf{x}) u_{n}^{(1)}(\mathbf{x})=W_{n}^{(1)} u_{n}^{(1)}(\mathbf{x}) \quad \mathbf{x} \in \omega_{1}, \quad u_{n}^{(1)}(\mathbf{x})=0 \quad \mathbf{x} \in \partial \omega_{1}$

and similarly for $u_{n}^{(2)}(\mathbf{x})$. Finally we build the two field operators

$$
\hat{\psi}^{(1,2)}(\mathbf{x})=\sum_{n} \hat{a}_{n}^{(1,2)} u_{n}^{(1,2)}(\mathbf{x}), \quad \text { where } \quad\left[\hat{a}_{n}^{(1,2)}, \hat{a}_{n^{\prime}}^{(1,2) \dagger}\right]_{ \pm}=\delta_{n, n^{\prime}}
$$

and the creation and destruction operators referring to the two regions $\omega_{1}$ and $\omega_{2}$ commute (or anticommute). Relevant variables $\hat{A}_{j}(\mathbf{x})$ which are generally given for the non confined fields in terms of $\hat{\psi}(\mathbf{x}), \hat{\psi}^{\dagger}(\mathbf{x})$ are now expressed in terms of $\hat{\psi}^{(1,2)}(\mathbf{x}), \hat{\psi}^{(1,2) \dagger}(\mathbf{x})$ according to $\mathbf{x} \in \omega_{1}$ or $\mathbf{x} \in \omega_{2}$. This seems to be the most straightforward way of partitioning the system into two non interacting parts and to represent the physical setup that is necessary for this: also different boundary conditions are conceivable, our choice represents the perfectly reflecting walls. In our point of view, putting confined systems in the foreground, boundary conditions have a very important role and unconfined field theory is only the starting point to introduce concrete descriptions. Since the Hamiltonian of a system extending on a region $\omega$ is given by $\hat{H}=\int_{\omega} d^{3} \mathbf{x} \hat{e}(\mathbf{x})$ one has $\hat{H}=\hat{H}^{(1)}+\hat{H}^{(2)}$ where $\hat{H}^{(1)}, \hat{H}^{(2)}$ is expressed only in terms of $\hat{a}_{n}^{(1)}, \hat{a}_{n}^{(1) \dagger}$ and $\hat{a}_{n}^{(2)}, \hat{a}_{n}^{(2) \dagger}$ respectively, so that $\left[\hat{H}^{(1)}, \hat{H}^{(2)}\right]=0$; furthermore, since $\hat{a}_{n}^{(1)}, \hat{a}_{n}^{(1) \dagger}$ and $\hat{a}_{n}^{(2)}, \hat{a}_{n}^{(2) \dagger}$ commute (or anticommute) all the relevant variables $\hat{A}_{j}(\mathbf{x})$ and $\hat{A}_{j}(\mathbf{x}, \tau)=e^{+\frac{i}{\hbar} \hat{H} \tau} \hat{A}_{j}(\mathbf{x}) e^{-\frac{i}{\hbar} \hat{H} \tau}$ have a tensor product structure:

$$
\int_{\omega_{1} \cup \omega_{2}} d^{3} \mathbf{x} \hat{A}_{j}(\mathbf{x}, \tau) \beta_{j}(\mathbf{x})=\int_{\omega_{1}} d^{3} \mathbf{x} \hat{A}_{j}^{(1)}(\mathbf{x}, \tau) \beta_{j}^{(1)}(\mathbf{x}) \otimes \hat{1}^{(2)}+\hat{1}^{(1)} \otimes \int_{\omega_{2}} d^{3} \mathbf{x} \hat{A}_{j}^{(2)}(\mathbf{x}, \tau) \beta_{j}^{(2)}(\mathbf{x})
$$


with $\beta_{j}^{(1)}(\mathbf{x})=\beta_{j}(\mathbf{x}), \mathbf{x} \in \omega_{1}, \beta_{j}^{(2)}(\mathbf{x})=\beta_{j}(\mathbf{x}), \mathbf{x} \in \omega_{2}$. If we assume that such a separation between system 1 and system 2 also occurs during the preparation time interval $\left[T, t_{0}\right]$, i.e., the operators $\hat{A}_{j}\left(\mathbf{x},-\left(t_{0}-t^{\prime}\right)\right)$ and $\hat{\mathbf{J}}_{j}\left(\mathbf{x},-\left(t_{0}-t^{\prime}\right)\right)$, $t^{\prime} \in\left[T, t_{0}\right]$ which appear in (1) also have the structure (2), one has the factorized structure

$$
\hat{\varrho}_{t_{0}}=\hat{\varrho}_{t_{0}}^{(1)} \otimes \hat{\varrho}_{t_{0}}^{(2)}
$$

with $\hat{\varrho}_{t_{0}}^{(1)}\left(\hat{\varrho}_{t_{0}}^{(2)}\right)$ having the structure $(1)$ with region $\omega$ replaced by $\omega_{1}\left(\omega_{2}\right)$. Obviously if this situation has been obtained by a previous preparation procedure implementing the separation, one has to assume that correlations between 1 and 2 are already decayed at time $T$. This kind of assumption is anyway necessary to start any description of isolated systems. Now we are interested in a more sophisticated situation: during the preparation time $\left[T, t_{0}\right]$ some very particular interaction arose, which eventually we will describe as due to a microsystem coming from system 2 and effecting system 1 at times $t>t_{0}$. In this way system 2 becomes a source and system 1 a detector for the microsystem. The difference in the procedure of isolating system 1 for times $t>t_{0}$ in the two cases: already isolated from part 2 during $\left[T, t_{0}\right]$ or interacting with part 2 during $\left[T, t_{0}\right]$, has relevant consequences on the dynamics of the system; in fact in the second case the time scale of the processes happening inside the detector should be taken into account. From a formal point of view in the new situation the time dependence of the operators in (1) should be ruled by an effective Hamiltonian, which no longer has the structure (2). Now our aim is not to give a detailed description of how the source works, but to indicate key points characterizing this kind of coupling and correlation between the two systems.

At time $t_{0}$ the statistical operator $\hat{\varrho}_{t_{0}}$ should be represented as:

$$
\hat{\varrho}_{t_{0}}=\frac{\exp \left\{-\left[\hat{S}_{t_{0}}^{(1)}+\hat{S}_{t_{0}}^{(2)}+\hat{C}_{t_{0}}^{(12)}\right]\right\}}{\operatorname{Tr}\left[\exp \left\{-\left[\hat{S}_{t_{0}}^{(1)}+\hat{S}_{t_{0}}^{(2)}+\hat{C}_{t_{0}}^{(12)}\right]\right\}\right]}
$$

where $\hat{C}_{t_{0}}^{(12)}$ is the part arising from the contribution of (1) related to the preparation in the time interval $\left[T, t_{0}\right]$ where operators $\hat{a}_{n}^{(1)}, \hat{a}_{n}^{(1) \dagger}$ and $\hat{a}_{n}^{(2)}, \hat{a}_{n}^{(2) \dagger}$ appear together. An expansion of $\hat{\varrho}_{t_{0}}$ with respect to $\hat{C}_{t_{0}}^{(12)}$ can be given, such that the basic positivity property of $\hat{\varrho}_{t_{0}}$ is granted. Starting from the representation:

$$
e^{\hat{A}+\hat{B}}=\left(\hat{1}+\int_{0}^{1} d u e^{u(\hat{A}+\hat{B})} \hat{B} e^{-u \hat{A}}\right) e^{\hat{A}}=e^{\hat{A}}\left(\hat{1}+\int_{0}^{1} d u e^{-u \hat{A}} \hat{B} e^{u(\hat{A}+\hat{B})}\right)
$$

one has the identity:

$e^{\hat{A}+\hat{B}}=e^{\frac{\hat{A}+\hat{B}}{2}} e^{\frac{\hat{A}+\hat{B}}{2}}=\left(\hat{1}+\int_{0}^{\frac{1}{2}} d u e^{u(\hat{A}+\hat{B})} \hat{B} e^{-u \hat{A}}\right) e^{\hat{A}}\left(\hat{1}+\int_{0}^{\frac{1}{2}} d u e^{-u \hat{A}} \hat{B} e^{u(\hat{A}+\hat{B})}\right)$ 
which leads to the following expansion with respect to $\hat{B}$ :

$$
e^{\hat{A}+\hat{B}}=\left(\hat{1}+\int_{0}^{\frac{1}{2}} d u e^{u \hat{A}} \hat{B} e^{-u \hat{A}}+\ldots\right) e^{\hat{A}}\left(\hat{1}+\int_{0}^{\frac{1}{2}} d u e^{-u \hat{A}} \hat{B} e^{u \hat{A}}+\ldots\right) .
$$

The first term of this kind of expansion gives an insight into the dynamics described by $\hat{\varrho}_{t_{0}}$, in fact setting

$$
\hat{\varrho}_{t_{0}}^{(1)}=\frac{\exp \left\{-\hat{S}_{t_{0}}^{(1)}\right\}}{\operatorname{Tr}\left[\exp \left\{-\hat{S}_{t_{0}}^{(1)}\right\}\right]}, \quad \hat{\varrho}_{t_{0}}^{(2)}=\frac{\exp \left\{-\hat{S}_{t_{0}}^{(2)}\right\}}{\operatorname{Tr}\left[\exp \left\{-\hat{S}_{t_{0}}^{(2)}\right\}\right]}
$$

and

$$
\hat{\mathbf{C}}_{t_{0}}^{(12)}=\int_{0}^{\frac{1}{2}} d u\left(\hat{\varrho}_{t_{0}}^{(1)} \otimes \hat{\varrho}_{t_{0}}^{(2)}\right)^{u} \hat{C}_{t_{0}}^{(12)}\left(\hat{\varrho}_{t_{0}}^{(1)} \otimes \hat{\varrho}_{t_{0}}^{(2)}\right)^{-u}
$$

one has:

$$
\hat{\varrho}_{t_{0}}=\frac{\left(\hat{1}+\hat{\mathbf{C}}_{t_{0}}^{(12)}\right) \hat{\varrho}_{t_{0}}^{(1)} \otimes \hat{\varrho}_{t_{0}}^{(2)}\left(\hat{1}+\hat{\mathbf{C}}_{t_{0}}^{(12) \dagger}\right)}{\operatorname{Tr}\left[\left(\hat{1}+\hat{\mathbf{C}}_{t_{0}}^{(12)}\right) \hat{\varrho}_{t_{0}}^{(1)} \otimes \hat{\varrho}_{t_{0}}^{(2)}\left(\hat{1}+\hat{\mathbf{C}}_{t_{0}}^{(12) \dagger}\right)\right]} .
$$

Let us consider

$\hat{N}_{1}=\int_{\omega_{1}} d^{3} \mathbf{x} \hat{\psi}^{(1) \dagger}(\mathbf{x}) \hat{\psi}^{(1)}(\mathbf{x})=\sum_{n} \hat{a}_{n}^{(1) \dagger} \hat{a}_{n}^{(1)}, \hat{N}_{2}=\int_{\omega_{2}} d^{3} \mathbf{x} \hat{\psi}^{(2) \dagger}(\mathbf{x}) \hat{\psi}^{(2)}(\mathbf{x})=\sum_{n} \hat{a}_{n}^{(2) \dagger} \hat{a}_{n}^{(2)}$.

The part of the operator $\hat{\mathbf{C}}_{t_{0}}^{(12)}$ that will have the most important role in our treatment has the form:

$$
\hat{\mathbf{C}}_{t_{0}}^{(12)}=\sum_{h} \hat{a}_{h}^{(1) \dagger} \hat{D}_{h}^{(2)}+\ldots
$$

where $\hat{D}_{h}^{(2)}$ indicates an operator built with $\hat{a}_{n}^{(2)}, \hat{a}_{n}^{(2) \dagger}$ having the role of a destruction operator in region 2 , i.e.:

$$
\hat{N}_{2} \hat{D}_{h}^{(2)}=\hat{D}_{h}^{(2)}\left(\hat{N}_{2}-1\right) .
$$

In fact in expression (4) only one creation operator $\hat{a}_{h}^{(1) \dagger}$ related to the modes of system 1 appears, so that the remaining part related to system 2 must have the role of a destruction operator for the system 2 , since

$$
\left[\hat{\mathbf{C}}_{t_{0}}^{(12)}, \hat{N}\right]=0,
$$

and all relevant variables in this non relativistic theory must commute with $\hat{N}=\hat{N}_{1}+\hat{N}_{2}$. Other terms would be:

$$
\sum_{h_{1}, h_{2}} \hat{a}_{h_{1}}^{(1) \dagger} \hat{a}_{h_{2}}^{(2) \dagger} \hat{D}_{h_{1}, h_{2}}^{(2)}, \quad \hat{N}_{2} \hat{D}_{h_{1}, h_{2}}^{(2)}=\hat{D}_{h_{1}, h_{2}}^{(2)}\left(\hat{N}_{2}-2\right),
$$


and similar ones. These kind of contributions would simply be associated in our treatment with a "two-particle" system, or more generally a compound microsystem. Also contributions in which the role of the indexes 1 and 2 is exchanged generally arise (however not necessarily since $\hat{\mathbf{C}}_{t_{0}}^{(12)}$, differently from $\hat{C}_{t_{0}}^{(12)}$, does not need to be self-adjoint), such terms would have a similar physical interpretation simply by interchanging the role of system 1 and system 2 . However also other terms generally appear together with contribution (4), e.g.

$\sum_{h_{1}, h_{2}, k} \hat{a}_{h_{1}}^{(1) \dagger} \hat{a}_{h_{2}}^{(1) \dagger} \hat{a}_{k}^{(1)} \hat{D}_{h_{1}, h_{2}, k}^{(2)}+\sum_{h_{1}, h_{2}, h_{3}, k_{1}, k_{2}} \hat{a}_{h_{1}}^{(1) \dagger} \hat{a}_{h_{2}}^{(1) \dagger} \hat{a}_{h_{3}}^{(1) \dagger} \hat{a}_{k_{1}}^{(1)} \hat{a}_{k_{2}}^{(1)} \hat{D}_{h_{1}, h_{2}, h_{3}, k_{1}, k_{2}}^{(2)}+\ldots$

In fact already due to the structure (3) of $\hat{\mathbf{C}}_{t_{0}}^{(12)}$ and the correlations among the modes $u_{n}^{(1)}$ in $\hat{\varrho}_{t_{0}}^{(1)}$ these terms would anyway arise in $\hat{\mathbf{C}}_{t_{0}}^{(12)}$. However we must expect that for meaningful preparation procedures operators $\hat{\mathbf{C}}_{t_{0}}^{(12)}$ generally arise whose contribution to the dynamics of relevant variables decays for time $t>$ $t_{0}+\tau, \tau$ being a microscopic correlation time: otherwise any treatment of nonequilibrium isolated systems would be impossible. Indeed the very scope of our article is to indicate a very particular situation in which this general assumption fails. So it seems reasonable to assume that the contribution of these terms to the dynamics of relevant variables can be neglected for $t \geq t_{0}+\tau$. Clearly this point requires further investigation. In conclusion we shall now investigate the dynamics of $\hat{\varrho}_{t}$ for $t \geq t_{0}+\tau$, taking simply

$$
\hat{\mathbf{C}}_{t_{0}}^{(12)}=\sum_{h} \hat{a}_{h}^{(1) \dagger} \hat{D}_{h}^{(2)}
$$

so that

$$
\hat{\varrho}_{t}=\frac{\mathcal{U}_{t-t_{0}}^{(1)} \otimes \mathcal{U}_{t-t_{0}}^{(2)}\left[\hat{1}+\sum_{h} \hat{a}_{h}^{(1) \dagger} \hat{D}_{h}^{(2)}\right] \hat{\varrho}_{t_{0}}^{(1)} \otimes \hat{\varrho}_{t_{0}}^{(2)}\left[\hat{1}+\sum_{k} \hat{a}_{k}^{(1)} \hat{D}_{k}^{(2) \dagger}\right] \mathcal{U}_{t-t_{0}}^{(1) \dagger} \otimes \mathcal{U}_{t-t_{0}}^{(2) \dagger}}{\operatorname{Tr}\left[\left[\hat{1}+\sum_{h} \hat{a}_{h}^{(1) \dagger} \hat{D}_{h}^{(2)}\right] \hat{\varrho}_{t_{0}}^{(1)} \otimes \hat{\varrho}_{t_{0}}^{(2)}\left[\hat{1}+\sum_{k} \hat{a}_{k}^{(1)} \hat{D}_{k}^{(2) \dagger}\right]\right]}
$$

for $t-t_{0}>\tau$. Let us stress that for all observables $\hat{A}=\hat{A}^{(1)} \otimes \hat{A}^{(2)}$ such that

$$
\left[\hat{A}^{(1)}, \hat{N}_{1}\right]=0, \quad\left[\hat{A}^{(2)}, \hat{N}_{2}\right]=0,
$$

and these are the only meaningful observables for systems 1 and $2, \hat{\varrho}_{t}$ is equivalent to the mixture

$$
\begin{aligned}
\hat{\varrho}_{t}= & \lambda \mathcal{U}_{t-t_{0}}^{(1)} \otimes \mathcal{U}_{t-t_{0}}^{(2)} \hat{\varrho}_{t_{0}}^{(1)} \otimes \hat{\varrho}_{t_{0}}^{(2)} \mathcal{U}_{t-t_{0}}^{(1) \dagger} \otimes \mathcal{U}_{t-t_{0}}^{(2) \dagger} \\
& +(1-\lambda) \frac{\mathcal{U}_{t-t_{0}}^{(1)} \otimes \mathcal{U}_{t-t_{0}}^{(2)} \sum_{h} \hat{a}_{h}^{(1) \dagger} \hat{D}_{h}^{(2)} \hat{\varrho}_{t_{0}}^{(1)} \otimes \hat{\varrho}_{t_{0}}^{(2)} \sum_{k} \hat{a}_{k}^{(1)} \hat{D}_{k}^{(2) \dagger} \mathcal{U}_{t-t_{0}}^{(1) \dagger} \otimes \mathcal{U}_{t-t_{0}}^{(2) \dagger}}{\operatorname{Tr}\left[\sum_{h} \hat{a}_{h}^{(1) \dagger} \hat{D}_{h}^{(2)} \hat{\varrho}_{t_{0}}^{(1)} \otimes \hat{\varrho}_{t_{0}}^{(2)} \sum_{k} \hat{a}_{k}^{(1)} \hat{D}_{k}^{(2) \dagger}\right]}
\end{aligned}
$$

where

$$
\lambda^{-1}=\operatorname{Tr}\left[\sum_{h} \hat{a}_{h}^{(1) \dagger} \hat{D}_{h}^{(2)} \hat{\varrho}_{t_{0}}^{(1)} \otimes \hat{\varrho}_{t_{0}}^{(2)} \sum_{k} \hat{a}_{k}^{(1)} \hat{D}_{k}^{(2) \dagger}\right] .
$$


In fact one has

$$
\operatorname{Tr}\left[\hat{A}^{(1)} \otimes \hat{A}^{(2)} \sum_{h} \hat{a}_{h}^{(1) \dagger} \hat{D}_{h}^{(2)} \hat{\varrho}_{t_{0}}^{(1)} \otimes \hat{\varrho}_{t_{0}}^{(2)}\right]=0,
$$

as can be seen indicating with $\left|N_{1}, N_{2}, S\right\rangle$ a basis of eigenstates of $\hat{N}_{1}, \hat{N}_{2}$, so that:

$$
\begin{aligned}
& \operatorname{Tr}\left[\hat{A}^{(1)} \otimes \hat{A}^{(2)} \sum_{h} \hat{a}_{h}^{(1) \dagger} \hat{D}_{h}^{(2)} \hat{\varrho}_{t_{0}}^{(1)} \otimes \hat{\varrho}_{t_{0}}^{(2)}\right]= \\
& \sum_{N_{1}, N_{2}, S}\left\langle N_{1}, N_{2}, S\left|\hat{A}^{(1)} \otimes \hat{A}^{(2)} \sum_{h} \hat{a}_{h}^{(1) \dagger} \hat{D}_{h}^{(2)} \hat{\varrho}_{t_{0}}^{(1)} \otimes \hat{\varrho}_{t_{0}}^{(2)}\right| N_{1}, N_{2}, S\right\rangle=0
\end{aligned}
$$

since due to (6) the state

$$
\hat{A}^{(1)} \otimes \hat{A}^{(2)} \sum_{h} \hat{a}_{h}^{(1) \dagger} \hat{D}_{h}^{(2)} \hat{\varrho}_{t_{0}}^{(1)} \otimes \hat{\varrho}_{t_{0}}^{(2)}\left|N_{1}, N_{2}, S\right\rangle
$$

belongs to the eigenstate of $\hat{N}_{1}, \hat{N}_{2}$ corresponding to eigenvalue $N_{1}+1, N_{2}-1$. Let us comment on the physical meaning of (7). Decomposing the expectations of the relevant variables according to this mixture one can associate to the two components of the mixture different state parameters $\zeta(t)$, different corresponding Gibbs states and different entropies. For the dynamics of the first component of the mixture, constructing in the usual way the non-equilibrium statistical operator, it is possible to assume that a "simple dynamics" arises, i.e., the memory term in the non-equilibrium statistical operator decays within a correlation time: then the usual methods of non-equilibrium statistical mechanics apply. If we had considered terms like (5) and also terms in which the role of indexes 1 and 2 is reversed, we would still obtain $\hat{\varrho}_{t}$ equivalent to a mixture of statistical operators corresponding to these different situations, with an obvious physical meaning. For the dynamics of the second component the typical structure of the non-equilibrium statistical operator is no longer feasible. We shall see that the dynamics of a "microsystem" becomes an important ingredient to describe what happens.

\section{Initial correlation and the appearance of a mi- crosystem}

The dynamics of the second component of the mixture (7) is given by

$$
\hat{\varrho}_{t}=\frac{\mathcal{U}_{t-t_{0}}^{(1)} \otimes \mathcal{U}_{t-t_{0}}^{(2)} \sum_{h} \hat{a}_{h}^{(1) \dagger} \hat{D}_{h}^{(2)} \hat{\varrho}_{t_{0}}^{(1)} \otimes \hat{\varrho}_{t_{0}}^{(2)} \sum_{k} \hat{a}_{k}^{(1)} \hat{D}_{k}^{(2) \dagger} \mathcal{U}_{t-t_{0}}^{(1) \dagger} \otimes \mathcal{U}_{t-t_{0}}^{(2) \dagger}}{\operatorname{Tr}\left[\sum_{h} \hat{a}_{h}^{(1) \dagger} \hat{D}_{h}^{(2)} \hat{\varrho}_{t_{0}}^{(1)} \otimes \hat{\varrho}_{t_{0}}^{(2)} \sum_{k} \hat{a}_{k}^{(1)} \hat{D}_{k}^{(2) \dagger}\right]}
$$




$$
=\frac{\sum_{h, k} \mathcal{U}_{t-t_{0}}^{(1)} \hat{a}_{h}^{(1) \dagger} \mathcal{U}_{t-t_{0}}^{(1) \dagger} \hat{\varrho}_{t}^{(1)} \mathcal{U}_{t-t_{0}}^{(1)} \hat{a}_{k}^{(1)} \mathcal{U}_{t-t_{0}}^{(1) \dagger} \otimes \mathcal{U}_{t-t_{0}}^{(2)} \hat{D}_{h}^{(2)} \hat{\varrho}_{t_{0}}^{(2)} \hat{D}_{k}^{(2) \dagger} \mathcal{U}_{t-t_{0}}^{(2) \dagger}}{\operatorname{Tr}\left[\sum_{h} \hat{a}_{h}^{(1) \dagger} \hat{D}_{h}^{(2)} \hat{\varrho}_{t_{0}}^{(1)} \otimes \hat{\varrho}_{t_{0}}^{(2)} \sum_{k} \hat{a}_{k}^{(1)} \hat{D}_{k}^{(2) \dagger}\right]} .
$$

According to the fact that we have chosen the term (4) to characterize the structure of $\hat{\mathbf{C}}_{t_{0}}^{(12)}$, we are now mainly interested in the dynamics of observables $\hat{A}^{(1)} \otimes \hat{1}^{(2)}$, then one has:

$$
\begin{aligned}
& \operatorname{Tr}\left[\hat{A}^{(1)} \otimes \hat{1}^{(2)} \hat{\varrho}_{t}\right]= \\
& \frac{\sum_{h, k} \operatorname{Tr}_{\mathcal{H}^{(1)}}\left[\hat{A}^{(1)} \mathcal{U}_{t-t_{0}}^{(1)} \hat{a}_{h}^{(1) \dagger} \mathcal{U}_{t-t_{0}}^{(1) \dagger} \hat{\varrho}_{t}^{(1)} \mathcal{U}_{t-t_{0}}^{(1)} \hat{a}_{k}^{(1)} \mathcal{U}_{t-t_{0}}^{(1) \dagger}\right] \operatorname{Tr}_{\mathcal{H}^{(2)}}\left[\hat{D}_{h}^{(2)} \hat{\varrho}_{t_{0}}^{(2)} \hat{D}_{k}^{(2) \dagger}\right]}{\sum_{h, k} \operatorname{Tr}_{\mathcal{H}^{(1)}}\left[\hat{a}_{h}^{(1) \dagger} \hat{\varrho}_{t_{0}}^{(1)} \hat{a}_{k}^{(1)}\right] \operatorname{Tr}_{\mathcal{H}^{(2)}}\left[\hat{D}_{h}^{(2)} \hat{\varrho}_{t_{0}}^{(2)} \hat{D}_{k}^{(2) \dagger}\right]}
\end{aligned}
$$

Let us introduce the following notations:

$$
\begin{aligned}
w_{h k}\left(t_{0}\right) & =\frac{\operatorname{Tr}_{\mathcal{H}^{(2)}}\left[\hat{D}_{h}^{(2)} \hat{\varrho}_{t_{0}}^{(2)} \hat{D}_{k}^{(2) \dagger}\right]}{\sum_{h} \operatorname{Tr}_{\mathcal{H}^{(2)}}\left[\hat{D}_{h}^{(2) \dagger} \hat{D}_{h}^{(2)} \hat{\varrho}_{t_{0}}^{(2)}\right]} \\
A_{k h}(t) & =\operatorname{Tr}_{\mathcal{H}^{(1)}}\left[\hat{A}^{(1)} \mathcal{U}_{t-t_{0}}^{(1)} \hat{a}_{h}^{(1) \dagger} \mathcal{U}_{t-t_{0}}^{(1) \dagger} \hat{\varrho}_{t}^{(1)} \mathcal{U}_{t-t_{0}}^{(1)} \hat{a}_{k}^{(1)} \mathcal{U}_{t-t_{0}}^{(1) \dagger}\right]
\end{aligned}
$$

then (9) can be written as

$\operatorname{Tr}\left[\hat{A}^{(1)} \otimes \hat{1}^{(2)} \hat{\varrho}_{t}\right]=\sum_{h, k} A_{k h}(t) w_{h k}\left(t_{0}\right) \frac{\sum_{h} \operatorname{Tr}_{\mathcal{H}^{(2)}}\left[\hat{D}_{h}^{(2) \dagger} \hat{D}_{h}^{(2)} \hat{\varrho}_{t_{0}}^{(2)}\right]}{\sum_{h, k} \operatorname{Tr}_{\mathcal{H}^{(1)}}\left[\hat{a}_{h}^{(1) \dagger} \hat{\varrho}_{t_{0}}^{(1)} \hat{a}_{k}^{(1)}\right] \operatorname{Tr}_{\mathcal{H}^{(2)}}\left[\hat{D}_{h}^{(2)} \hat{\varrho}_{t_{0}}^{(2)} \hat{D}_{k}^{(2) \dagger}\right]}$

and hints towards the typical structure of one-particle quantum mechanics. To $w_{h k}\left(t_{0}\right)$ the role can be given of the statistical operator at time $t_{0}$ for a oneparticle system, when one takes as a basis to represent it the normal modes of system 1 in $L^{2}\left(\omega_{1}\right) ; A_{k h}(t)$ can be seen as the representative of an observable, embodying time dependence. Obviously we will have to check this idea looking at the dynamics of the system: in this way the correlation between system 1 and system 2 should be explained in terms of a particle produced by system 2 and detected by system 1 . However all this holds if the additional correlation described by the numerical factor

$$
\sigma=\frac{\sum_{h} \operatorname{Tr}_{\mathcal{H}^{(2)}}\left[\hat{D}_{h}^{(2) \dagger} \hat{D}_{h}^{(2)} \hat{\varrho}_{t_{0}}^{(2)}\right]}{\sum_{h, k} \operatorname{Tr}_{\mathcal{H}^{(1)}}\left[\hat{a}_{h}^{(1) \dagger} \hat{\varrho}_{t_{0}}^{(1)} \hat{a}_{k}^{(1)}\right] \operatorname{Tr}_{\mathcal{H}^{(2)}}\left[\hat{D}_{h}^{(2)} \hat{\varrho}_{t_{0}}^{(2)} \hat{D}_{k}^{(2) \dagger}\right]}
$$

can be neglected. We shall see that by rather natural assumptions this factor is practically one. In fact let us diagonalize the matrix $w_{h k}\left(t_{0}\right)$

$$
w_{h k}\left(t_{0}\right)=\sum_{\alpha} \lambda^{\alpha}\left(t_{0}\right)\left\langle h \mid \psi_{0}^{\alpha}\right\rangle\left\langle\psi_{0}^{\alpha} \mid k\right\rangle, \quad \lambda^{\alpha}\left(t_{0}\right)>0, \quad \sum_{\alpha} \lambda^{\alpha}\left(t_{0}\right)=1 ;
$$


then:

$\frac{1}{\sigma}=\sum_{h, k, \alpha} \operatorname{Tr}_{\mathcal{H}^{(1)}}\left[\hat{a}_{h}^{(1) \dagger} \hat{\varrho}_{t_{0}}^{(1)} \hat{a}_{k}^{(1)}\right]\left\langle h \mid \psi_{0}^{\alpha}\right\rangle\left\langle\psi_{0}^{\alpha} \mid k\right\rangle \lambda^{\alpha}\left(t_{0}\right)=\sum_{\alpha} \operatorname{Tr}_{\mathcal{H}^{(1)}}\left[\hat{a}_{\psi_{0}^{\alpha}}^{(1) \dagger} \hat{\varrho}_{0}^{(1)} \hat{a}_{\psi_{0}^{\alpha}}^{(1)}\right] \lambda^{\alpha}\left(t_{0}\right)$

with

$$
\hat{a}_{\psi_{0}^{\alpha}}^{(1)}=\sum_{k} \hat{a}_{k}^{(1)}\left\langle\psi_{0}^{\alpha} \mid k\right\rangle
$$

where $\hat{a}_{\psi_{0}^{\alpha}}^{(1)}$ can be considered as the annihilation operator of a particle in the state $\sum_{h} u_{h}(\mathbf{x})\left\langle h \mid \psi_{0}^{\alpha}\right\rangle$. Let us assume for the moment that the statistical operator $w\left(t_{0}\right)$ is a pure state $w\left(t_{0}\right)=\left|\psi_{0}\right\rangle\left\langle\psi_{0}\right|$, then:

$$
\frac{1}{\sigma}=\operatorname{Tr}_{\mathcal{H}^{(1)}}\left[\hat{a}_{\psi_{0}}^{(1) \dagger} \hat{\varrho}_{t_{0}}^{(1)} \hat{a}_{\psi_{0}}^{(1)}\right]=1 \pm \operatorname{Tr}_{\mathcal{H}^{(1)}}\left[\hat{a}_{\psi_{0}}^{(1) \dagger} \hat{a}_{\psi_{0}}^{(1)} \hat{\varrho}_{t_{0}}^{(1)}\right]
$$

with $\hat{a}_{\psi_{0}}^{(1)}=\sum_{k} \hat{a}_{k}^{(1)}\left\langle\psi_{0} \mid k\right\rangle$. Taking into account that system 1 should have the role of a detection device for the microsystem it is rather natural to assume that this one-particle state $\left|\psi_{0}\right\rangle$ is very scarcely occupied, i.e.:

$$
\operatorname{Tr}_{\mathcal{H}^{(1)}}\left[\hat{a}_{\psi_{0}}^{(1) \dagger} \hat{a}_{\psi_{0}}^{(1)} \hat{\varrho}_{t_{0}}^{(1)}\right] \ll 1
$$

and as a consequence $\sigma \approx 1$. More generally we shall assume that $w\left(t_{0}\right)$ is a mixture of one-particle states $\left\{\left|\psi_{0}^{\alpha}\right\rangle \alpha=1,2, \ldots, n\right\}$ that are scarcely occupied. Let us stress that it is just the skill of the experimentalist to build sources that prepare possibly pure states. In this situation (10) becomes:

$$
\operatorname{Tr}\left[\hat{A}^{(1)} \otimes \hat{1}^{(2)} \hat{\varrho}_{t}\right]=\sum_{h, k} A_{k h}(t) w_{h k}\left(t_{0}\right)=\operatorname{Tr}_{L^{2}\left(\omega_{1}\right)}\left[A(t) w\left(t_{0}\right)\right]
$$

where in the last term the trace refers to the one-particle Hilbert space in which the operators $A(t), w\left(t_{0}\right)$ are represented. Let us observe that the correspondence from $\hat{A}^{(1)}$ to $A(t)$ has the following properties: $\hat{1}^{(1)}$ is transferred to the identity on $L^{2}\left(\omega_{1}\right)$, positivity is preserved and therefore a projection valued or p.o.v. measure $\hat{E}^{(1)}$ is transferred to a p.o.v. measure on $L^{2}\left(\omega_{1}\right)$. Let us consider a little further the dynamics of the macrosystem 1 referring to the subcollection that has to be demixed at time $t_{0}+\tau$ and described by the statistical operator (8). Let us restrict for simplicity to the case in which the source prepares a pure state $\psi_{0}$. Then one has from (10) the following expression for expectations of the observables of system 1 at a time $t>t_{0}+\tau$ :

$$
\left\langle\hat{A}^{(1)}\right\rangle_{t}=\operatorname{Tr}_{\mathcal{H}^{(1)}}\left[\hat{A}^{(1)} \mathcal{U}_{t-t_{0}}^{(1)} \hat{a}_{\psi_{0}}^{\dagger} \mathcal{U}_{t-t_{0}}^{(1) \dagger} \hat{\varrho}_{t}^{(1)} \mathcal{U}_{t-t_{0}}^{(1)} \hat{a}_{\psi_{0}} \mathcal{U}_{t-t_{0}}^{(1) \dagger}\right]=\operatorname{Tr}_{\mathcal{H}^{(1)}}\left[\hat{A}^{(1)}(t) \hat{\varrho}_{t}^{(1)}\right]
$$

where

$$
\hat{A}^{(1)}(t)=\hat{a}_{\psi_{0}}(t) \hat{A}^{(1)} \hat{a}_{\psi_{0}}^{\dagger}(t)
$$


with

$$
\hat{a}_{\psi_{0}}(t)=\mathcal{U}_{t-t_{0}}^{(1)} \hat{a}_{\psi_{0}}^{\dagger} \mathcal{U}_{t-t_{0}}^{(1) \dagger}
$$

Then one is led to a redefinition of the relevant observables for the macrosystem influenced by the microsystem

$$
\hat{A}_{j}^{(1)}(\mathbf{x}) \rightarrow \hat{\mathbf{A}}_{j}^{(1)}(\mathbf{x})
$$

$\hat{\mathbf{A}}_{j}^{(1)}(\mathbf{x})=\hat{a}_{\psi_{0}}(t) \hat{A}_{j}^{(1)}(\mathbf{x}) \hat{a}_{\psi_{0}}^{\dagger}(t)=\hat{A}_{j}^{(1)}(\mathbf{x})+\left[\hat{a}_{\psi_{0}}(t), \hat{A}_{j}^{(1)}(\mathbf{x})\right] \hat{a}_{\psi_{0}}^{\dagger}(t) \pm \hat{A}_{j}^{(1)}(\mathbf{x}) \hat{a}_{\psi_{0}}^{\dagger}(t) \hat{a}_{\psi_{0}}(t)$

and one considers a new reference Gibbs state related to $\hat{\mathbf{A}}_{j}(\mathbf{x})$ and the corresponding state parameters. Then one can study the dynamics of $\hat{\varrho}_{t}^{(1)}$ writing it as a new equilibrium statistical operator for $t>t_{0}+\tau$ and a decisive simplification would occur if its dynamics should be "simple". We can fairly well expect that this is the case. The time dependence of $\hat{a}_{\psi_{0}}(t)$ is linked to the dynamics of the microsystem as we shall discuss in the next sections: it will even give an insight into the physics of the microsystem. Here we want to stress how the concept of microsystem was helpful to face the problem of a non simple dynamics of a macrosystem that we have here demixed into two possibly simple dynamics. Let us observe that the time dependence of the expectations of observables of system 1 is twofold: it arises once from $\hat{a}_{\psi_{0}}(t)$ and is related to the physics of the microsystem, it arises further from $\hat{\varrho}_{t}^{(1)}$ and is related to the general dynamics of the macrosystem; one can easily characterize variables that are scarcely effected by the microsystem. Let us consider $\hat{A}_{j}^{(1)}(\mathbf{x})$ such that

$$
\left[\hat{a}_{\psi_{0}}(t), \hat{A}_{j}^{(1)}(\mathbf{x})\right]=0,
$$

furthermore one can expect that

$$
\operatorname{Tr}\left[\hat{A}_{j}^{(1)}(\mathbf{x}) \hat{a}_{\psi_{0}}^{\dagger}(t) \hat{a}_{\psi_{0}}(t) \hat{\varrho}_{t}^{(1)}\right] \ll \operatorname{Tr}\left[\hat{A}_{j}^{(1)}(\mathbf{x}) \hat{\varrho}_{t}^{(1)}\right]
$$

in fact it was assumed by (12) that the state $\psi_{0}$ was depleted for $\hat{\varrho}_{t_{0}}^{(1)}$, so that this inequality should hold at time $t=t_{0}$ and one can expect that it also holds during the time evolution of the microsystem in absence of too strong interactions with the macrosystem. Then for these $\hat{A}_{j}(\mathbf{x})$ one has by (13)

$$
\left\langle\hat{\mathbf{A}}_{j}^{(1)}(\mathbf{x})\right\rangle_{t}=\operatorname{Tr}_{\mathcal{H}^{(1)}}\left[\hat{A}_{j}^{(1)}(\mathbf{x}) \hat{\varrho}_{t}^{(1)}\right]
$$

and no dynamical consequence due to the microsystem arises. On the contrary if by the choice of an observable $\hat{A}_{j}^{(1)}(\mathbf{x})$ one meets the situation $\left[\hat{a}_{\psi_{0}}(t), \hat{A}_{j}(\mathbf{x})\right] \neq$ 0 , one expects that by a suitable $\hat{\varrho}_{\bar{t}}^{(1)}$ a significant macroscopic signal can arise: in this way the dynamics of the microsystem in the time interval $\left[t_{0}, t\right]$ can be tested. 


\section{Dynamics of the microsystem}

Let us first make the simple assumption that the interaction of a particle created by $\hat{a}_{\psi_{0}}^{\dagger}$ with the different modes of the macrosystem can be neglected, then one has immediately by (11)

$\hat{a}_{\psi_{0}}^{\dagger}(t)=\mathcal{U}_{t-t_{0}} \hat{a}_{\psi_{0}}^{\dagger} \mathcal{U}_{t-t_{0}}^{\dagger}=\mathcal{U}_{t-t_{0}} \sum_{h} \hat{a}_{h}^{\dagger}\left\langle h \mid \psi_{0}\right\rangle \mathcal{U}_{t-t_{0}}^{\dagger}=\sum_{h} e^{-\frac{i}{\hbar} W_{h}\left(t-t_{0}\right)} \hat{a}_{h}^{\dagger}\left\langle h \mid \psi_{0}\right\rangle=\hat{a}_{\psi_{t}}^{\dagger}$

where $\psi_{t}(\mathbf{x})=\sum_{h} u_{h}(\mathbf{x}) e^{-\frac{i}{\hbar} W_{h}\left(t-t_{0}\right)}\left\langle h \mid \psi_{0}\right\rangle$ is a one-particle wave function, determined by the coefficients $\left\langle h \mid \psi_{0}\right\rangle$ which are given by (11) and were obtained studying the source part related to system 2. $\psi_{t}(\mathbf{x})$ satisfies the Schrödinger equation which rules the normal modes of the Schrödinger field: then oneparticle quantum mechanics is extracted from the formalism. Of course this is only an approximation: the microsystem interacts with the normal modes of the macrosystem and the general problem of decoherence is now met [7]. Let us stress how naturally this phenomenon appears: one can hardly talk about a microsystem without taking it into account. The problem of studying the dynamics of a microsystem in the present context coincides with the well-known general problem of deriving master equations. It appears here that the investigation of structures of the kind $\hat{a}_{\psi_{0}}^{\dagger}(t) \hat{a}_{\psi_{0}}(t), \psi_{0}$ involving the normal modes of a macrosystem is a very natural starting point to derive master equations [8]. As a consequence of interactions other modes of the system are involved. One expects that at least for $t-t_{0}$ short enough a mixture of states $\psi_{t}^{\alpha}$ replaces the pure state $\psi_{t}$, the one-particle system associated to it being no longer isolated, such that

$$
\operatorname{Tr}\left[\hat{a}_{\psi_{t}^{\alpha}}^{\dagger} \hat{a}_{\psi_{t}^{\alpha}} \hat{\varrho}_{t}^{(1)}\right] \ll 1
$$

then the concept makes sense of binary "collisions" between these one-particle states $\psi_{t}$ and the feeded normal modes of the system. This situation is formally the same as the case of a particle undergoing Brownian motion inside a macroscopic system; models for such a case have been recently discussed inside many-body scattering theory [9]. Extending slightly such a situation one expects that the expression $\sum_{\alpha} \lambda^{\alpha}(t) \hat{a}_{\psi_{t}^{\alpha}}^{\dagger} \hat{a}_{\psi_{t}^{\alpha}}$ is obtained from $\hat{a}_{\psi_{0}}^{\dagger} \hat{a}_{\psi_{0}}$ by a linear map with a generalized Lindblad structure. Then decoherence is made explicit and related to the properties of the macroscopic system: this non Hamiltonian dynamics already spoils the universal features of the dynamics of the microsystem. At sufficiently longer times, as an increasing set of states $\psi_{t}^{\alpha}$ is involved, there is no more a distinction between the one-particle state and the other modes of the system. Before this happens however the quantum behavior of the microsystem had an essential role inside the dynamics of the macroscopic system.

A final comment is in order now: we have seen that one reaches aspects of quantum mechanics of a microsystem starting from a given macrosystem. However in this way only a very particular sector of the physics of the microsystem is used and explored; i.e., a particular state $\psi_{0}$ is prepared, a not too long time 
evolution of it is allowed, only some particular macroscopic variables can be effected by the microsystem. The one-particle Hilbert space which comes into evidence refers to the particular confinement region $\omega_{1}$. The point is that this treatment encompasses all possible macrosystems fitting in our model. Then the picture of a microsystem arises taking into account all possible settings. Finally it is an idealization that must be compatible with all of them: it is only at this stage that the usual connection of microsystem with symmetry properties makes sense. Endorsing this point of view microsystems are at the root of any local process underlying the physics of all macrosystems; the classical idea that these are structures composed of microsystems can be taken on in quantum mechanics as a meaningful model only if localization of microsystems in phase-space need not be better than inside regions of value $\gg \hbar^{3}$ : then as it is well-known a phase-space density of particles, building the macrosystem, can be introduced by means of a Wigner function related to $\hat{\varrho}_{t}$.

\section{Acknowledgments}

L. Lanz and B. Vacchini gratefully acknowledge financial support by MURST under Cofinanziamento. B. Vacchini also acknowledges support by MURST under Progetto Giovani.

\section{References}

[1] Proceedings of the $3^{\text {rd }}$ Workshop on Mysteries, Puzzles and Paradoxes in Quantum Mechanics, R. Bonifacio, B. G. Englert and D. Vitali Eds., Z. Naturforsch. 56a, (2001).

[2] D. N. Zubarev, Non-equilibrium statistical thermodynamics (Consultant Bureau, New York, 1974); D. N. Zubarev, V. Morozov and G. Roepke, Statistical mechanics of non-equilibrium processes (Akademie-Verlag, Berlin, 1996).

[3] G. Ludwig, An Axiomatic Basis for Quantum Mechanics (Springer, Berlin, 1985); Foundations of Quantum Mechanics (Springer, Berlin, 1983).

[4] A. Barchielli, L. Lanz, G. M. Prosperi, Nuovo Cimento 72B, 79 (1982); Found. Phys. 13, 779 (1983); E. B. Davies, Quantum Theory of Open Systems (Academic Press, London, 1976); K. Kraus, States, Effects and Operations, Lecture Notes in Physics, Vol. 190, (Springer, Berlin, 1983); P. Busch, M. Grabowski and P. J. Lahti, Operational Quantum Physics, Lecture Notes in Physics, Vol. m31, (Springer, Berlin, 1995). 
[5] A. S. Holevo, Probabilistic and Statistical Aspects of Quantum Theory (North Holland, Amsterdam, 1983); Statistical Structure of Quantum Theory, Lecture Notes in Physics, Vol. m67, (Springer, Berlin, 2001).

[6] L. Lanz, O. Melsheimer and B. Vacchini, Rep. Math. Phys. 46, 191 (2000); J. Mod. Opt. 47, 2165 (2000).

[7] D. Giulini, E. Joos, C. Kiefer, J. Kupsch, I.-O. Stamatescu, and H. D. Zeh, Decoherence and the Appearance of a Classical World in Quantum Theory (Springer, Berlin, 1996); Decoherence: Theoretical, Experimental, and Conceptual Problems, Ph. Blanchard, D. Giulini, E. Joos, C. Kiefer and I.-O. Stamatescu Eds., Lecture Notes in Physics, Vol. 538, (Springer, Berlin, 1998).

[8] L. Lanz and B. Vacchini, Int. J. Theor. Phys. 36, 67 (1997); Phys. Rev. A 56, 4826 (1997); Int. J. Theor. Phys. 37, 545 (1998); B. Vacchini, Int. J. Theor. Phys. 39, 927 (2000).

[9] B. Vacchini, Phys. Rev. Lett. 84, 1374 (2000); R. F. O'Connell, Phys. Rev. Lett. 87, 028901 (2001); B. Vacchini, Phys. Rev. Lett. 87, 028902 (2001); Phys. Rev. E 63, 066115 (2001); Z. Naturforsch. 56a, 230 (2001); J. Math. Phys. 42, 4291 (2001); L. Lanz and B. Vacchini, Int. J. Mod. Phys. 36, 435 (2002); B. Vacchini, Int. J. Theor. Phys. (to appear). 